\title{
Design Blades of a Wind Turbine Using Flexible Multibody Modelling
}

\author{
Maria Augusta Neto ${ }^{1}$, Wenbin Yu ${ }^{2}$, Jorge A.C. Ambrósio ${ }^{3}$ \\ ${ }^{1}$ Faculdade de Ciência e Tecnologia da Universidade de Coimbra (Polo II), 3020 Coimbra, Portugal. \\ ${ }^{2}$ Utah State University, Logan, UT 84322-4130, USA \\ ${ }^{3}$ Instituto Superior Técnico, Av. Rovisco Pais, 1041-001, Lisboa, Portugal.
}

\begin{abstract}
A methodology for the application of structural optimization to find the optimal layouts of fiber composite blades used on the multibody model of a wind turbine is presented. The VABS (the Variational Asymptotic Beam Section analysis) methodology is used to compute the Timoshenko-like stiffness associated with the blade sectional properties. The gravity and the inertia effects are taking in account as well as the aerodynamic force, which are calculated by the aerodynamic coefficients and transferred automatically to the corresponding finite element nodes. The goal of the optimization process is to improve the twist-flap coupling and, simultaneously, to fulfill a set of multidisciplinary constraints. Considering this requirements, the optimal values for the fiber orientation of the composite spar of the blade must be determined. These methodologies rely on the efficient and accurate calculation of the system sensitivities to support the optimization algorithms. In this work a general formulation for the computation of the first order analytical sensitivities based on the direct method is used. The direct method for sensitivity calculation is obtained by differentiating the equations defining the response of the structure with respect the design variables. The equations of motion and sensitivities of the flexible multibody system are solved simultaneously and therefore the accelerations and velocities of the system and the sensitivities of the accelerations and of the velocities are integrated in time using a multi-step multi-order integration algorithm.
\end{abstract}

\section{Introduction}

The design of composite rotor blades in modern industrial environments requires complex and accurate computational models, which may restrict design exploration and prevent designers from try innovative layups and cross sectional configurations. Therefore, the variations of the cross sectional layouts across the wide range of modern rotor blades are remarkable small compared to the wealth of possibilities provided by the utilization of composite materials $^{1}$ (Volvoi, et al., 2004). Composite blades are the type of structures encountered in helicopter rotor blades and in wind turbines blades ${ }^{2}$ (Hodges and $\mathrm{Yu}, 2007$ ).

The wind turbine modeling complexity relates the tools used in the construction of the models of individual and coupled components of the technical systems. Because of the special cross section geometric feature of rotor blades the use of tools that provide a fast and accurate mean of assessing the cross section blade mechanical properties opens an opportunity to improve the design cycle of composite rotor blades. The VABS routines library (Variational Asymptotic Beam Section analysis) allow modeling nonhomogeneous anisotropic beams with arbitrary cross section geometries using a 2-D finite discretization of the beam cross section and, therefore, permit a high-fidelity representation of the blade structure in terms of an 
equivalent beam model ${ }^{3}$ (Yu, et al., 2002). Once the equivalent cross sectional stiffness is obtained, every beam finite element analyses can be carry out. The outline of the blade used in this work is a SERI-8 aerofoil with the chord length of $594 \mathrm{~mm}^{4}$ (Ong and Tsai, 2000).

To reduce the transient loads of wind turbine rotor blades, the aeroelastic tailoring concept has been used since the late 1980s. Using this concept the blade geometry and/or structure can be designed such that when the blade undergoes flapwise deflection during operation, it will inherently undergo twist as well. Two basic approaches used are either: 1) to sweep the blade in the plane of rotation, allowing that the aerodynamic loading acting on outboard portions of the blade produces a substantial torque about the blade elastic axis, or 2) rotate some of the structural laminate fibers away from the spanwise direction inducing, in this way, a twist-flap coupling in the structure ${ }^{5}$ (Wetzel, 2005). Within the optimization of flexible multibody systems the second approach is used, in this paper, to investigate for the optimal off-axis fiber orientation on the spar cap that maximize the magnitude of twist-flap coupling.

A general approach for sensitivity analysis of rigid-flexible multibody systems with composite materials is used in the optimization procedure. Since the design variables used are the layer orientation of some laminates that make up the blades cross section, the constitutive relation sensitivities are computed by extending the VABS routines library to evaluate these new matrix quantities, which take in account the warping sensitivities with respect to the design variables. The sensitivities of the equations of motion are analytically formulated and implemented using the direct differentiation method. The dynamic equations and the time derivatives of the sensitivities are all integrated at the same time, thus the control of the time integration errors become more effective.

The optimization of the multibody composite components is performed by taking the ply orientations of lamination as continuous design variables. The rigid-flexible multibody dynamics code and the sensitivity analysis code are linked with general optimization algorithms included in the package DOT/DOC ${ }^{6}$ (Vanderplaats, 1992).

\section{References}

${ }^{1}$ Volvoi, V.V., Yoon, S., Lee, C.-Y. and Hodges,D.H., 2004. "Structural Optimization of Composite Rotor Blades”, $45^{\text {th }}$ AIAA/ASME/ASCE/AHS/ASC Structures, Structural Dynamics \& Materials Conference, 19-22 April 2004, Palm Springs, California, USA.

${ }^{2}$ Hodges D.H. and Yu, W., 2007. "A Rigorous, Engineer-friendly Approach for Modelling Realistic Composite Rotor Blades”, Wind Energy, 10, pp. 179-193.

${ }^{3} \mathrm{Yu}$, W., Hodges, D.W., Volvoi, V. and Cesnik, C.E.S., 2002. "On Timoshenko-like modelling of initially curved and twisted Composite beams", International Journal of Solids and Structures, 39, pp. 5101-5121

${ }^{4}$ Ong, C.-H. and Tsai, S.W., 2000. "The Use of Carbon Fibers in Wind Turbine Blade Design,” SAND2000-0478, Sandia National Laboratories, Albuquerque, New Mexico, USA.

${ }^{5}$ Wetzel, K.K., 2005“Utility Scale Twist-Flap Coupled Blade Design”, Journal of Solar Energy Engineering, 127, pp. 529-537.

${ }^{6}$ Vanderplaats, G., 1992. DOT-Design Optimization Tools, Version 3.0 VMA Engineering , Colorado springs, USA. 